\title{
Synthesis and Application of Carbon Matrix Composites
}

\author{
Jianqing Qi, Weiping Tao, Yong Wang, Guanwu Xue \\ Department of Materials Engineering and Applied Chemistry, Sanya University of Science and Technology, Hainan, \\ China
}

\begin{abstract}
This paper describes the development of carbon-based composites, and the synthesis and application of four carbonbased composites of graphene, carbon nanotubes, fullerenes and graphite.

Keywords: carbon-based composites, graphene, carbon nanotubes, fullerenes, graphite alkynes

Received: Nov 22, 2018 Accepted: Dec 25, 2018 Online: Jan 12, 2019

\section{Introduction}

Composite material is composed of two or more different substances through a certain way to combine together to form another multi-phase material. Generally the composite material is continuous, called the matrix, while the other phase is dispersed, called the reinforcement material. The composite material concentrates on the unique advantages of the substrate and the reinforcing material.
\end{abstract}

As the name suggests, carbon-based composite (CBC) material has a carbon matrix, which is usually carbon fiber (fabric) or silicon carbide, and is reinforced with other materials. The reinforcing agent may still be carbon but in a different from the matrix. It is a highly thermal-stable material developed in the 1960s. Carbon composites maintain the advantages of carbon (graphite) and have (i) greatly improved toughness and strength, (ii) reduced thermal expansion coefficient, (iii) high chemical and thermal stability (non-oxidation Sexual atmosphere), (iv) good thermal conductivity and (v) good corrosion resistance.

CBCs have the sum advantage of two or more composites, which are superior to any single-texture material. Therefore, they have a wider range of applications, which also give rise to a strong interest in CBCs. In recent years, the discovery of new carbon materials represented by graphene and nitrogen-doped graphene has made carbon materials a star with a unique nature and a wide range of applications.

\subsection{Development}

Carbon / carbon composites came out about 1958. Initially, the experimental staff of the Chancewater aircraft company's aerospace branch were accidentally discovered due to an accident when studying phenolic resin-based composites. Meanwhile, the United States Union Carbide Corporation (UCC) with graphite cloth reinforced resin, after curing, carbonization and graphitization, made the first batch of carbon / carbon composite materials and sold them as commodity.

The development of carbon / carbon composites represented by the United States can be roughly divided into three phases: The initial stage: From 1958 to mid-1960s. This stage featured the basic research work on the carbon / CBC materials, for example, to find the technical key of carbon fiber elastic modulus and strength. In 1965, chemical vapor deposition was introduced to the synthesis process.

The second stage: From mid-1960s to the early 1970s, during which carbon / CBC began their application. A great deal of work was done on the enhanced carbon, matrix carbon and composite processes. Carbon / CBCs had been 
used as a thermal-stable material for cutting-edge technology such as rocket engine nozzles, satellites and spacecraft.

The third stage: Since the early 1970s until now. The properties of CBCs were further improved, the anisotropy was successfully solved, and the three-dimensional orthogonal were developed. Their application (e.g. anti-thermal shock effects) and microstructures were studied. Also in this stage they were used as a strategic nuclear weapon head heatresistant material. MK-12A carbon / carbon nose was successfully used as the third-generation intercontinental missile Warheads.

\subsection{Preparation}

Dipping method: That is, with the impregnated molten oil or coal tar pitc, and then by carbonation and graphite treatment. Its matrix is graphite carbon was layered structure, the performance is anisotropic. There are also impregnat- ed with thermosetting resin such as furfuryl alcohol or phenolic resin which is treated only by carbonization. Its matrix is glassy carbon, that is, amorphous carbon structure and the performance is isotropic.

CVD method: That is, the pyrolytic carbon of the hydrocarbon compound is deposited on the reinforcement to compound, and the carbon matrix of this method is pyrolytic carbon similar to glassy carbon. Carbon / carbon composites are not resistant to oxidation and sometimes need to add antioxidant coating.

\section{Graphene}

The hybrid form of the carbon atom in the graphene is $\mathrm{sp}^{2}$, and the covalent bond with an angle of 120 degrees is connected with the other carbon atoms into a hexagonal layered honeycomb structure. In addition, each carbon atom has a pz. The number of coordination atoms in the layer is 3, and the bond length between adjacent carbon atoms is $0.142 \mathrm{~nm}$.

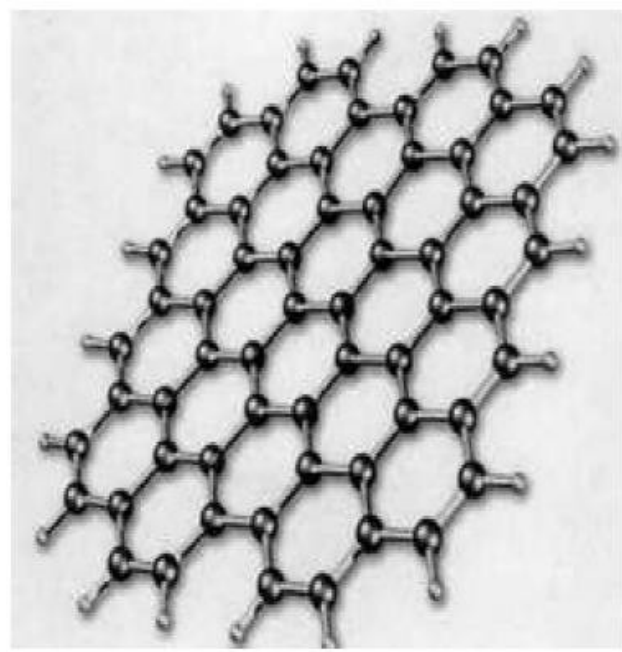

Graphene is the base of zero-dimensional fullerenes, one-dimensional carbon nanotubes and three-dimensional graphite. If the five-membered ring in the graphene plane lattice will bend the graphene plane, it will form a richler when there are more than 12 rings. Carbon nanotubes can be seen as crusted by graphene, and graphite can be seen as composed of graphene through the van der Waals stack.

\subsection{Synthesis of graphene}

Graphene has many excellent physical properties, for how large-scale, high-quality preparation of monolayer graphene is now scientists face an important research content. The main methods for preparing graphene so far are: mechanical stripping, chemical vapor deposition, epitaxial growth, chemical oxidation and reduction and other methods ${ }^{[1]}$.

\subsubsection{Mechanical stripping method ${ }^{[1-3]}$}

The mechanical stripping method was developed by the Geim Professor Group at the University of Manchester in the United Kingdom in 2004 to develop a graphene method. They use a transparent tape repeatedly paste the graphite, and then with a graphite sheet of adhesive tape affixed to the substrate such as silicon, and then dissolved with acetone tape 
and then monolayer and less graphene transferred to the substrate such as silicon ${ }^{[2]}$.

In addition to the use of micro-mechanical stripping method for the preparation of graphene, there is a more mature mechanical stripping method - liquid phase ultrasonic stripping method. Liquid phase separation method refers to the bulk of graphite placed in organic solvents such as N-methyl pyrrolidone (NMP) or o-dichlorobenzene for ultrasound, monolithic and multi-layer can be obtained in the coexistence of graphene dispersion ${ }^{[1]}$.

\subsubsection{Chemical Vapor Deposition ${ }^{[4]}$}

Chemical vapor deposition (CVD) principle is in the high temperature decomposition of gas $\left(\mathrm{CH}_{4}, \mathrm{C}_{2} \mathrm{H}_{2}\right)$, through high temperature annealing makes carbon atoms deposited on the surface of the substrate to form a single layer of graphene. This method is controllable and can produce graphene in large area.

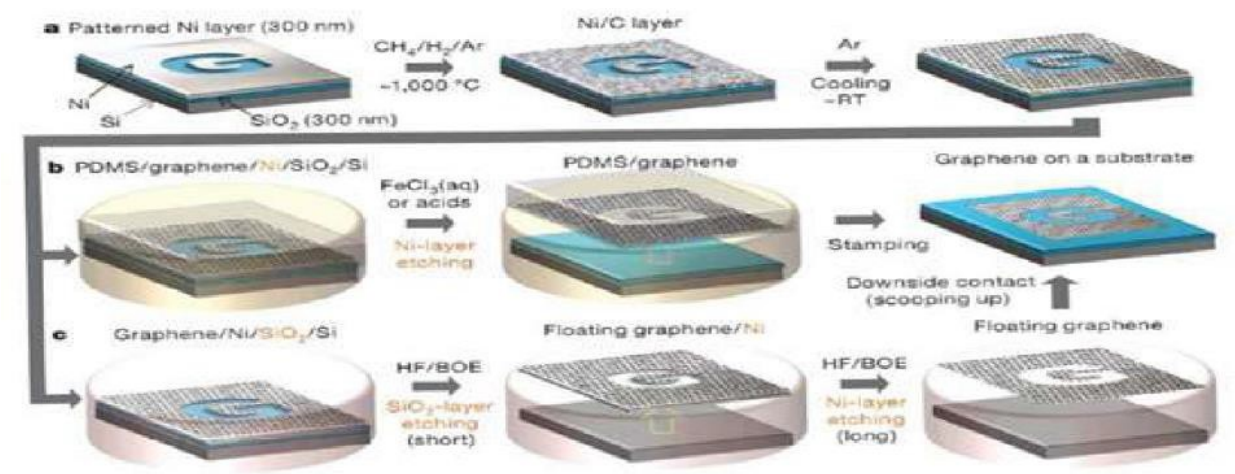

\subsubsection{Epitaxial growth method ${ }^{[5,6]}$}

Epitaxial growth means that the silicon is removed by high temperature heating under ultra-vacuum conditions, and the carbon atoms are rearranged to form a graphene sheet.

Heer et al. ${ }^{[7]}$ used silicon carbide as the raw material to evaporate silicon atoms from silicon carbide at $1300{ }^{\circ} \mathrm{C}$ and $1.33 \times 10^{-10} \mathrm{~Pa}$ high vacuum to obtain a continuous large area of graphene film. The epitaxial growth method can produce high quality graphene, but there are still some problems in controlling the number of layers and large area of graphene.

\subsubsection{Chemical oxidation reduction method ${ }^{[3]}$}

The Reduction of Graphene Oxide Solution is the most widely used method for the preparation of graphene. The method is cheap and the preparation process is simple. Chemical oxidation reduction method for the preparation of graphene has three processes: graphite oxidation $\rightarrow$ stripping $\rightarrow$ reduction, as shown below.

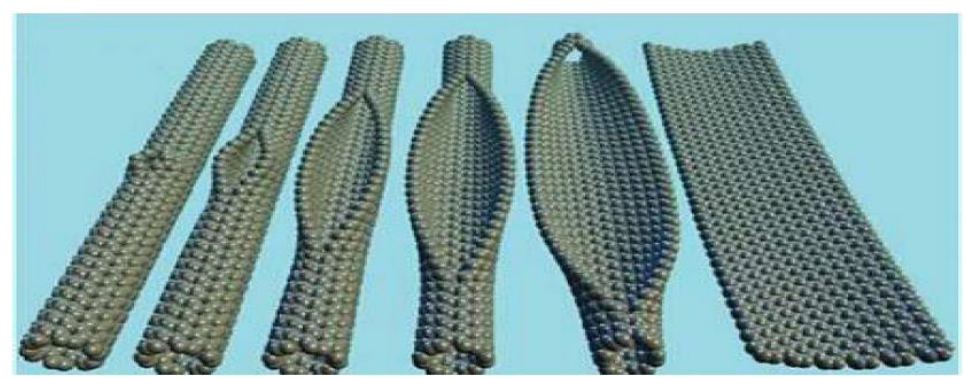

\subsubsection{Preparation of graphene nanobelts from carbon nanotubes ${ }^{[1]}$}

Due to the limited band gap of two-dimensional graphene, the application in many fields such as field-effect transistors is limited. It is found that quasi-one-dimensional graphene nanobelts (GNRs) have bandgap. The GNRs can be prepared by ultrasonic or chemical shear of two-dimensional graphene sheets, but the nanobelts obtained are not controllable ${ }^{[8]}$. Dai and so on through the chemical etching technology to cut MWNTs successfully prepared GNRs, first WMNTs deposited on the Si matrix, and then coated with a layer of MWNTs $300 \mathrm{~nm}$ PMMA film, followed by the use of Ar plasma etching MWNTs by controlling the etching Time to control the number of GNRs, and finally remove the polymer components to obtain GNRs with a width of 10-20 nm. The GNRs obtained have semiconductor 
properties and have a wide range of applications in the electronics industry. James and so on using potassium permanganate and sulfuric acid as oxidant, in the more moderate conditions along an axis gradually cut MWNTs and also obtained GNRs. The preparation of the schematic and mechanism shown in the following figure. Although GNRs prepared by oxidation are not semiconductors, this method is simpler and more efficient and easier to produce on a large scale.

\subsection{Application of graphene}

\subsubsection{Transparent conductive electrode}

At present, the most commonly used material for transparent conductive electrodes is indium doped tin oxide (ITO). ITO is widely used with mobile phone touch panels, monitors and solar cells, but indium is expensive for precious metals and difficult to recover. The graphene has the advantages of light weight, high strength, excellent flexibility and conductivity. At the same time, because of the special structure of graphene has a higher light transmission, quickly become the ideal material instead of ITO ${ }^{[3]}$.

Wang et al. used graphene as a transparent conductive film in a solar cell with a conductivity of $550 \mathrm{~S} / \mathrm{m}$ and a conversion efficiency of $0.26 \%$. Liu's Group made graphene electrode by reduction and repair of graphene oxide, the conductivity reached $350-410 \mathrm{~S} / \mathrm{cm}$, and the light transmittance was $95 \%$. Geng et al. prepared a transparent graphene conductive film by a two-step reduction method and applied it to an organic solar cell. The efficiency of the whole device was about $1.01 \%$.

\subsubsection{Super Capacitors ${ }^{[3,9,10]}$}

Super capacitor is between the capacitor and the battery between the energy storage device, is the transmission of energy and energy storage system. The specific surface area of graphene is very large and the conductivity is excellent, and graphene is stable in high conductivity while ensuring the sweep voltage. The large specific surface area of graphene can increase the aggregation capacity of electrons and can be used as the capacity of supercapacitors. The use of graphene as an electrode greatly reduces the charging time and increases the capacity by 5-6 times.
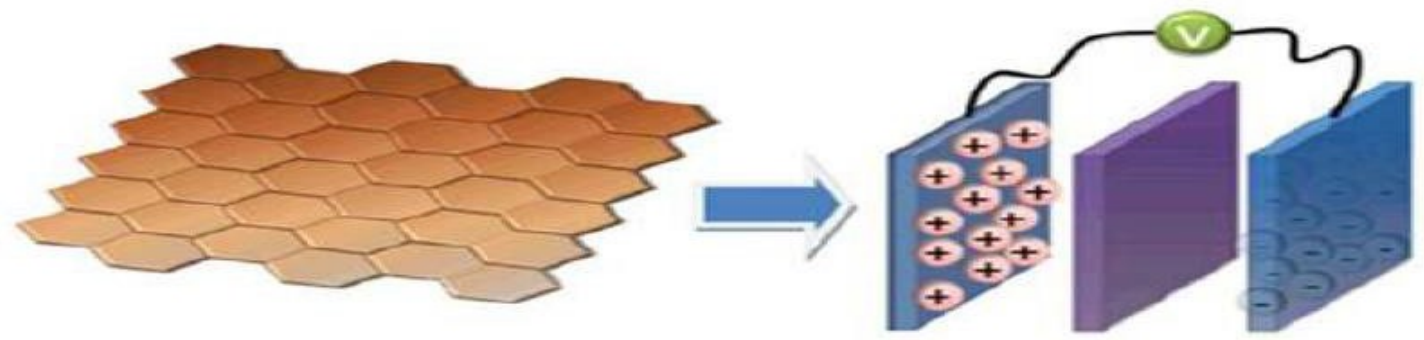

Due to the special structure of graphene, the researchers have done an in-depth study of supercapacitors. Stoller et al. used monolayer graphene as the electrode for supercapacitors, which had a specific capacitance of $135 \mathrm{~F} / \mathrm{g}$ in water and a specific capacitance of $99 \mathrm{~F} / \mathrm{g}$ in the organic electrolyte. Le et al. used a new method of directing the graphene solution onto the titanium mesh, and then preparing a supercapacitor of graphene film by thermal reduction. After 1000 cycles of voltammetry, the specific capacitance decreased from 125 to $121 \mathrm{~F} / \mathrm{g}$, and the loss rate was less than $3 \%$. Ning et al. prepared a controlled structure of graphene web by template chemical deposition method. The thickness of graphene web was 1-2 layers and the specific surface area was $1654 \mathrm{~m}^{2} / \mathrm{g}$. The polystyrene net was maintained at $94.1 \%$ after the 2000 volt cycle with a specific capacitance of $245 \mathrm{~F} / \mathrm{g}$ in the $\mathrm{KOH}$ solution as a supercapacitor electrode. Yoo et al. prepared a graphene-based planar structure of supercapacitors, the mass ratio of the capacitor can reach $250 \mathrm{~F} / \mathrm{g}$ while the area than the previous reported capacitance reached $394 \mathrm{~F} / \mathrm{cm}^{2}$.

\subsubsection{Sensor ${ }^{[3]}$}

The carbon atoms in the graphene are very exible to each other and form a large $\pi$-bond in the honeycomb. When strongly applied to the surface of the graphene, the surface of the graphene will bend, but the carbon atoms of the graphene surface do not need to be rearranged to accommodate the effects of external forces. And the structure of the gra- phene makes the graphene excellent in electrical conductivity. As shown below, for the graphene gas sensor. 


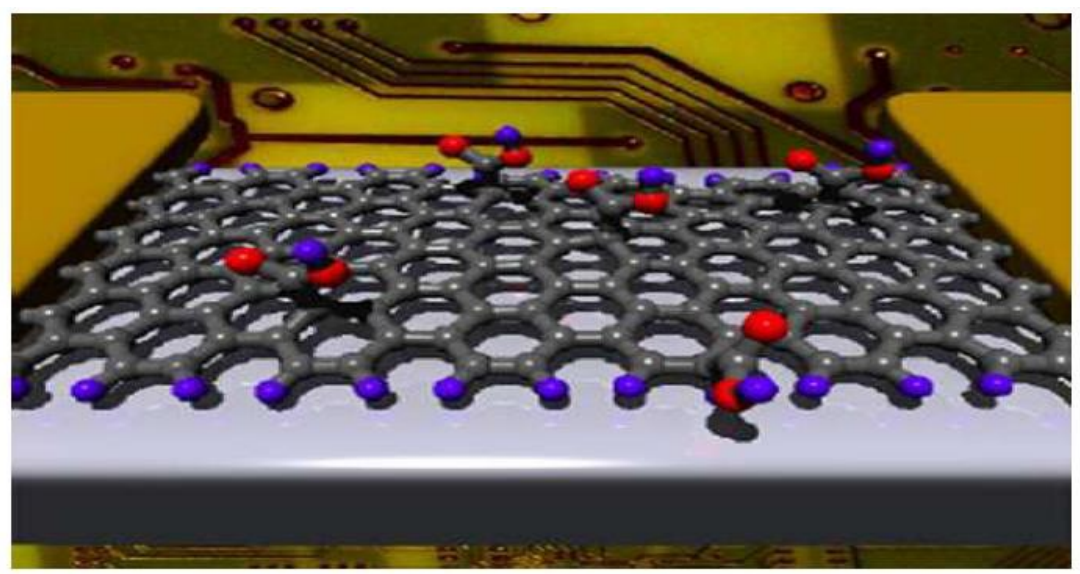

In 2007, Professor Novoselov et al. successfully prepared a graphene-based sensor with high sensor sensitivity to test the resistivity in a short time. $\left(\mathrm{CO}, \mathrm{NH}_{3}, \mathrm{NO}_{2}\right)$ adsorbed on graphene for effective research. Fowler et al. used highquality graphene by oxidation-reduction method, and successfully prepared a graphene-based sensor. In addition to gas detection, but also on the dinitrotoluene for effective detection. Zhang et al. found that doping graphene, in the preparation of sensors will improve their own sensitivity and select the accuracy. In the process of graphene preparation by chemical vapor deposition on the surface modification of Pd particles, the sensor of the $\mathrm{H}_{2}$ detection sensitivity greatly improved.

\section{Carbon nanotubes}

The carbon atoms in the carbon nanotubes are mainly sp2, but the cylindrical bending structure leads to quantum confinement and $\sigma$ - $\Pi$ re-hybridization, where the three $\sigma$ bonds are slightly deviated from the plane and the off -bound $\Pi$ orbit is more biased. The carbon nanotubes are divided into single-walled carbon nanotubes (SWNTs) and multiwalled carbon nanotubes (MWNTs).

\subsection{Synthesis of Carbon Nanotubes ${ }^{[11]}$}

At present, the preparation of single-walled carbon nanotubes has only yield levels, and it is still difficult to control single-walled carbon nanotubes with the desired structure. In contrast, the preparation of multi-walled carbon nanotubes is more mature, yield up to $\mathrm{kg} / \mathrm{h}$, and can control the diameter and growth orientation of carbon nanotubes. Arc method, laser ablation method, vapor deposition method is the more widely used method.

\subsubsection{Arc method:}

Laboratory arc synthesis device is generally in the lower than the atmospheric pressure Ar gas water cooled cavity, the horizontal or vertical placed two diameter of 6-12 mm, the gap of 1-4 mm graphite rod for the electrode, the DC discharge between the electrodes Arc. The carbon nanotubes produced by the arc process are less and less defective than the carbon nanotubes produced by other methods, and therefore have higher mechanical strength and better thermal conductivity.

The preparation of single-walled carbon nanotubes by arc method requires the drilling of an axial hole in the graphite rods filled with dense metal and graphite mixtures as the anode. There are a variety of metal or mixture catalysts to fill graphite rods, but nickel / yttrium and cobalt / nickel catalysts are more commonly used.

\subsubsection{Laser ablation method:}

Laser ablation is an effective method for the preparation of single - walled carbon nanotubes. It is a metal catalyst and graphite mixed graphite target placed in the middle of the quartz tube, the tube is placed in a furnace heating. When the furnace temperature rises to a certain temperature (such as $1473 \mathrm{~K}$ ), through the inert gas, and a laser beam focused on the graphite target. The graphite target generates gaseous carbon under the irradiation of the laser, and the gaseous carbon and the catalyst particles are transported from the high temperature zone to the low temperature region by the gas stream to produce carbon nanotubes under the action of the catalyst. 


\subsubsection{Chemical Vapor Deposition:}

The carbonaceous gas is cracked by a heating or inductance heater, a high temperature furnace or an infrared lamp and a plasma, and the carbon nanotubes are grown on a substrate containing catalyst particles such as $\mathrm{Fe}, \mathrm{Co}$ and $\mathrm{Ni}$. The method has the advantages of low cost, large output and easy control of the experimental conditions. It is the most promising method for preparing high quality multiwalled carbon nanotubes.

In the later study, it was found that the carbon nanotubes were grown at the top or bottom of the carbon nanotubes, and two kinds of carbon nanotube growth models were proposed: bottom growth and top growth. The free carbon produced by the reaction system dissolves and diffuses in the catalyst particles, and when saturated, the carbon precipitates in the form of tubes. If the adhesion of the catalyst particles to the surface of the substrate is relatively strong, the carbon will precipitate from the upper surface of the particles, i.e. the bottom growth model. If the adhesion of the catalyst particles to the matrix is weak, the carbon will precipitate from the lower surface of the particles and the catalyst particles continue to improve, that is, the top growth model.

\subsubsection{Catalytic / reduced pyrolysis in confined systems:}

The carbon nanotubes are usually synthesized by catalytic / reducing pyrolytic organic molecules at 400-700 / C using an autoclave as the reaction vessel. This method is simple, easy to operate.

\subsection{Application of carbon nanotubes ${ }^{[6,11]}$}

\subsubsection{Field emission}

Carbon nanotubes have been shown to have good field emission properties, and field emission phenomena can be observed at an electric field intensity of less than $1 \mathrm{v} / \mu \mathrm{m}$, and the current intensity can reach $1 \mathrm{~A} / \mathrm{cm}^{2}$. According to the nature of carbon nanotubes, micro-arrangement, preparation process and the structure requirements of the transmitter, carbon nanotube field emitters can be made into a variety of structural forms. However, the possible application of single carbon nanotube field emission devices is limited to systems that require very high sharpness to electron sources, such as high resolution electron microscopy and lithographic techniques. In contrast, carbon nanotube film has a great potential for technical applications, it has a friendly launch performance, and easy to prepare and mass production. The field transmitter in the vacuum electronic devices, microwave amplifiers, field emission displays and X-ray tube and other fields has a very broad application prospects.

\subsubsection{Sensing}

Carbon nanotubes are unique one-dimensional quantum wires and have a very high surface-to-volume ratio, so their electrical properties are very sensitive to molecular adsorption. Especially in semiconducting single-walled carbon nanotubes, all carbon atoms are exposed to the surface so that a very small fraction of the charge generated by molecular adsorption is sufficient to allow the local carrier to run out, resulting in a large conductance change. Based on this, people have built a carbon nanotube-based sensing elements and converters for the detection of gas molecules and bio- molecules. Such as HJ. Dai and so on the use of polyethylene imide coated carbon tube developed a highly sensitive, highly selective carbon nanotube sensor. Woollcy et al reported the use of single-walled carbon nanotubes AFM tip directly produced kilobase DNA unit type method, so that people more deeply understand the gene on cancer and heart disease and other common diseases.

\subsubsection{Electrochemistry}

The diameter of the carbon nanotubes is at the nanometer scale, the spacing between the wall layers is $0.34 \mathrm{~nm}$, and the kinetic diameter of the $\mathrm{H}_{2}$ molecules is $0.289 \mathrm{~nm}$. Therefore, the hollow cavity and the bundle gap of the singlewalled carbon nanotubes are theoretically and interlayer gap can be used as hydrogen absorption position. Therefore, carbon nanotubes hydrogen storage research has aroused widespread concern. A.C. Dllfon and so on using the program temperature desorption method to infer the diameter of $1.2 \mathrm{~nm}, 100 \%$ purity $100 \%$ single-walled carbon tube hydrogen storage capacity of up to $5-10 \mathrm{wt} \%$. Rchen and so on through the multi-walled carbon nanotubes doped with metal $\mathrm{Li}$ and $\mathrm{K}$ will increase the hydrogen storage capacity to $20-14 \mathrm{wt} \%$, but its chemical stability and hydrogen evolution temperature is still subject to the actual conditions. 


\section{Fullerenes}

The hybridization of carbon atoms in fullerenes is between $\mathrm{sp} 2$ and sp3. The earliest discovered and representative structure of the fullerenes is the $\mathrm{C} 60$, which is a 20 -sided body made up of 20 six-membered rings and 12 fivemembered rings. The diameter is $0.7 \mathrm{~nm}$.

Fullerene is a thermodynamically unstable but thermodynamically stable metastable substance in carbon. From the energy point of view, the energy of the carbon atoms in the graphite is zero, and the energy of the carbon atoms in the fullerenes is as high as $0.45 \mathrm{eV}$. Therefore, it is necessary to apply graphite to fullerenes to exert very high energy from the outside.

\subsection{Synthesis of fullerenes ${ }^{[11]}$}

\subsubsection{Carbon evaporation method:}

The carbon atoms are evaporated in the inert gas atmosphere by means of arc, resistance heating, electron beam irradiation, laser evaporation, vacuum heat treatment, plasma and solar energy in the form of artificial / natural graphite or high carbon content coal Ambient pressure and the presence of different types of metal catalysts, the carbon atoms after evaporation are again clustered to form fullerenes.

\subsubsection{Catalytic pyrolysis Carbon-containing gases, hydrocarbons and organic compounds:}

$\mathrm{Fe}, \mathrm{Co}, \mathrm{Ni}$ and other metals as a catalyst, through the disproportionation of $\mathrm{CO}, \mathrm{C}_{2} \mathrm{H}_{2}$ or propylene and other gas phase cracking to synthesis. In addition, ferrocene and other organometallic compounds can be directly pyrolysis to produce fullerenes.

\subsubsection{Benz flame combustion method:}

In the flame temperature $1800 \mathrm{~K}$ under the conditions of benzene diluted by hydrogen after combustion to $\mathrm{C} 60$ and $\mathrm{C} 70$, this method is very effective for the preparation of $\mathrm{C} 70$, and easy to industrial production.

\subsubsection{Conversion of carbon-containing inorganic materials:}

At the substrate temperature of 600 / C conditions, by laser irradiation in the crystallization of SiC inside the larger size, less defective fullerenes.

\subsection{Application of fullerenes}

\subsubsection{Superconductor material}

The C60 molecule itself is a nonconductive insulator, but when the alkali metal is embedded with the void, the C60 and alkali metal series compounds will be converted to superconductors such as the K3C60 with a high superconducting critical temperature. Compared with the oxide superconductor, C60 series superconductor has a perfect three-dimensional superconductivity, current density, high stability, easy to develop wire and other advantages, is a very valuable new superconducting material.

\subsubsection{New polymer materials}

Due to the special cage structure and function of $\mathrm{C} 60$, a new functional polymer material with excellent conductivity and optical properties is obtained by introducing the polymer system into a novel functional group. Wang reported that the mixture of $\mathrm{C} 60$ / C70 was incorporated into the luminescent polymer material of polyvinyl carbazole (PVK) to obtain a new type of polymer photoconductor, its photoconductivity can be comparable with some of the best light guide materials. This kind of light guide material in the electrostatic copy, electrostatic imaging and light detection technology has a wide range of applications.

\subsubsection{Other aspects of application}

C60F60 as a 'molecular ball' and 'molecular lubricant' in high-tech plays an important role. Lithium atoms embedded in the carbon cage may be made of high-performance lithium battery. Carbon cage embedded in rare earth elements europium may become a new type of rare earth luminescent materials. Water-soluble C60 derivatives are expected to 
be used as new nuclear magnetic contrast agents. C60 and its derivatives may become novel catalysts and novel nanoscale molecular wires, molecular pipettes and whisker reinforced composites. The water-soluble host-guest complexes of $\mathrm{C} 60$ with cyclodextrin and cyclic aromatics will play an important role in supramolecular chemistry and biomimetic chemistry.

\section{Graphite alkyne}

Graphite is a kind of carbon isomorphic form formed by sp and sp2 and sp3. The benzene ring is conjugated by 1,3diyne bond and has a two-dimensional planar network structure. The special structure of the graphite alkyne makes it rich in carbon chemical bonds because the conjugated system is large and has a wide pitch of $4.1913 \AA$, and the graphite alkyne has good chemical stability and exhibits semiconductor properties.

\subsection{Synthesis of graphite alkyne ${ }^{[12]}$}

A wide range of graphene films were successfully synthesized on the surface of copper alloy by hexadecylbenzene catalyzed cross-coupling reaction on copper films. The films were highly ordered, with fewer defects and $10^{-3}$ to $10^{-4} \mathrm{~S}$ $/ \mathrm{m}$.

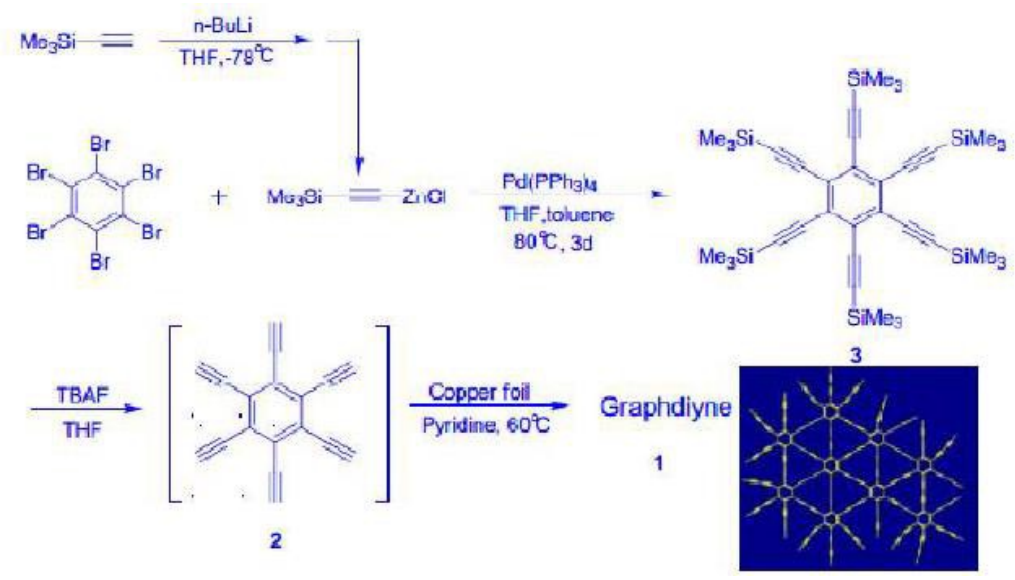

\subsection{Application of graphite alkyne ${ }^{[13-15]}$}

Du et al. will be doped with graphite acetylene nano-tablets in the organic solar cells within the anode, to enhance the photoelectric conversion efficiency of the battery. In the field of photocatalysis, Wang et al. reported a new type of photocatalyst of graphite-acetylene composite P25, which catalyzes the degradation of methylene blue (MB) by the composite catalyst is superior to the traditional P25 and graphene composite P25 catalyst.

\subsubsection{Gas separation}

The graphite alkyne has a rich pore structure that makes it an ideal molecular sieve for the realization of various gas separation needs. For example, it can be used as an ultra-thin separation membrane for $\mathrm{H}_{2}$ separation in $\mathrm{H}_{2}$ (mixed between $\mathrm{H}_{2}, \mathrm{CH}_{4}$ and $\mathrm{CO}$ ) -hydrogen. Purified potential application materials.

\subsubsection{Desalination}

Desalination of seawater using a graphite-based nano-porous membrane is widely considered to be an energy-efficient method that may outperform existing commercial technologies such as reverse osmosis. Buehler et al. found that graphite alkyne voids allow water molecules to permeate without barriers and completely reject salt ions through the carbon 'nanotubes', an excellent feature that is ideal for achieving desalination. Carbon nanotubes can be made from monolayer films of primary graphite alkynes, which are two-dimensional carbon allotropes. Graphite acetylene is highly inert, stable and porous has definite triangular atomic pores and single-atom thick mesh fibers structure.

\subsubsection{Hydrogen storage materials}

Metal doped graphite alkyne has excellent properties. Because of its additional in-plane $\pi$-conjugation that does not possess the sp2 graphene and fullerenes, the graphite alkyne exhibits enhanced binding energy to the calcium, so that they can be optimized for $\mathrm{H}_{2}$ storage materials. Liu and other predicted lithium-modified graphite alkyne can also be used as excellent hydrogen storage materials, the hydrogen storage capacity of up to $18.6 \%$. 
According to the order of the dielectric layer of the photonic crystal, it can be divided into one-dimensional, twodimensional and three-dimensional photonic crystals. The spatial structure of the one-dimensional photonic crystal was showed as Figure 1 below:

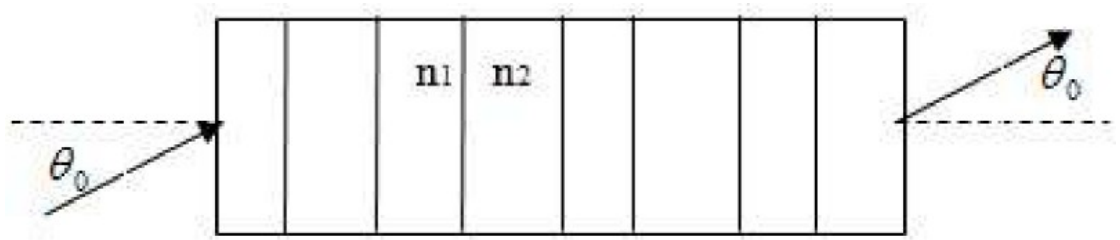

Figure 1. Spatial structure of one-dimensional photonic crystals

One-dimensional photonic crystal is a photonic crystal material with the dielectric refractive index in which it only periodically distributed in one direction in the space. The simplest one-dimensional photonic crystals are typically composed of two layers of dielectric thin films with different refractive indices. The one-dimensional photonic crystals cause the dielectric constant of which perpendicular to the dielectric layer to change periodically with respect to the spatial position, and the dielectric constant of which parallel to the direction of the plane does not change with the spatial position. One-dimensional photonic crystal structure is relatively simple, easy to prepare, easy to study, and it is a strong representation, and the application is very extensive, such as the antisense or permeable membrane in Fabry-Perot cavity optical multilayer.

Two-dimensional photonic crystals refer to materials with photonic bandgap properties in all directions of the twodimensional space, which are arranged in parallel and evenly by a plurality of dielectric rods. The basic structure of a common two-dimensional photonic crystal is shown in Figure 2, usually a regular crystal system formed by a dielectric column.
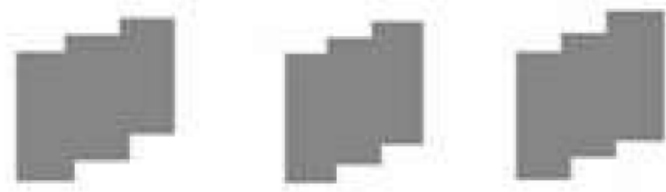

Figure 2. Spatial structure of two-dimensional photonic crystals

The dielectric constant of two-dimensional photonic crystal is changing periodically with the spatial position in which it is perpendicular to the medium direction (in both directions), and when parallel to the direction of dielectric rod, it does not change with the spatial position. In order to achieve the regulation of the bandgap range of the twodimensional photonic crystal frequency, the crystal structure composed of the array of dielectric rods can be changed to make the cross-section in a variety of shapes, such as rectangular, triangular, graphite hexagon and so on.

The common structure of the three-dimensional photonic crystal is the spatial periodic structure of the two media cubes, as shown in Figure 3:

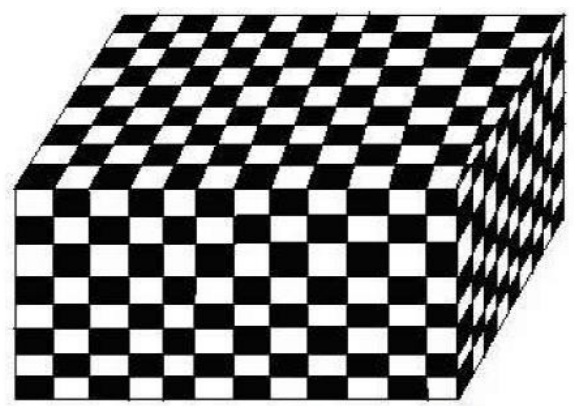

Figure 3. Spatial structure of three-dimensional photonic crystals

Three-dimensional photonic crystal materials have photonic frequency bandgap properties in three-dimensional space, and three-dimensional photonic crystals have a wide application prospect. Due to the limitation in science and technology, the preparation of three-dimensional photonic crystals is more difficult. The first three-dimensional 
photonic crystal with completed photonic bandgap is created by Yablonovitch of Bell Communications Research, a space periodic structure composed of many face-centered cubes, also known as diamond structures.

\section{Photonic crystal characteristics and application}

After the emerged of photonic crystals, it become very popular in optical research within a very short period of time. The most important and fundamental feature of photonic crystals is the band gap and conduction band. The study of the bandgap of photonic crystals can control the propagation state of light and suppress spontaneous radiation. For the doped photonic crystal, the defect mode can produce special characteristic such as photonic localization and others.

For the study of the band gap of photonic crystals, we must first understand the basic value of light propagation, that is, transmittance $\mathrm{T}$. The transmittance $\mathrm{T}$ for a single layer of media can be defined as follows: The transmittance is the ratio of the intensity of the emitted light to the incident light after passing through the medium. (As shown in Figure 4).

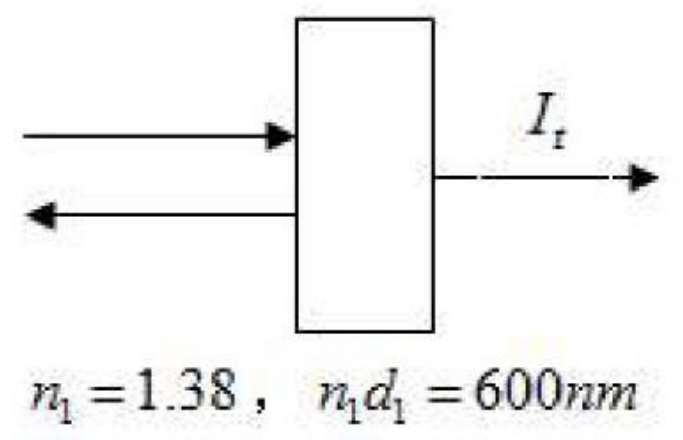

Figure 4.

Based on the conservation law, we know that the reflectivity $\mathrm{R}$ is in the above formula, $\mathrm{R}$ is the reflectance of the medium and $\mathrm{T}$ is the transmittance. Figure below (Figure.5) showed the transmission of a single layer of medium for different wavelengths of light. We define the range of transmittance $\mathrm{T}=0$ as the band gap in the photonic crystal, and the transmittance $>0$ is the conduction band of the photonic crystal. Usually photonic crystals are more likely to be applied on the absolute regulation of light, thus this paper also focuses on the band gap of photonic crystals, that is, the part that photons cannot be propagated.

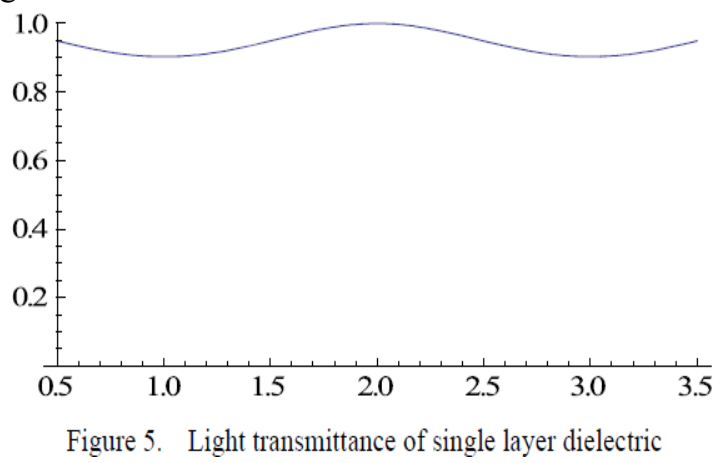

The study result showed the transmittance of one-dimensional photonic crystals as shown in Figure 6 below:

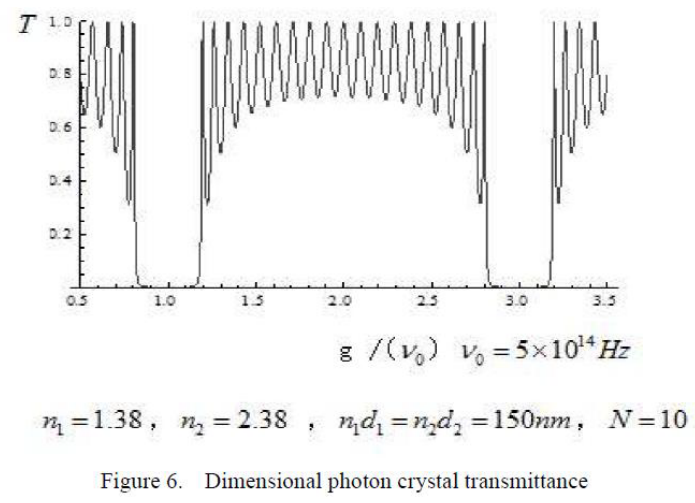


Through the application of photonic crystals, we can artificially control the propagation of electromagnetic waves (elastic waves), while band gap of photonic crystals can be used to develop the anti-electromagnetic radiation film. In one-dimensional photonic crystals, the characteristics of one-dimensional doping photonic crystals are also special. The basic structure shown in Figure 7:

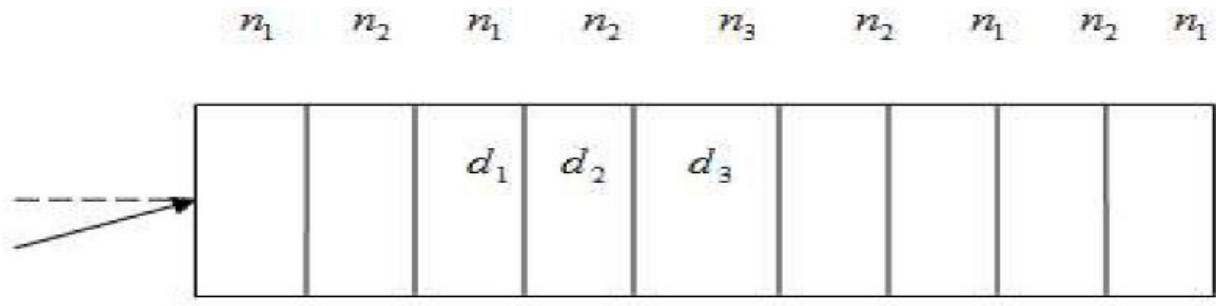

Figure 7. One - Dimensional Photonic Crystals

The addition of a third medium to a regular one-dimensional photonic crystal can form a one-dimensional doped photonic crystal, while keeping most of the properties of a one-dimensional photonic crystal, it is also showing a new characteristic, which is the defect mode. Figure 8 and Figure 9 show the TE wave and TM wave defect modes with the changes of incident angle and incident wavelength, respectively.
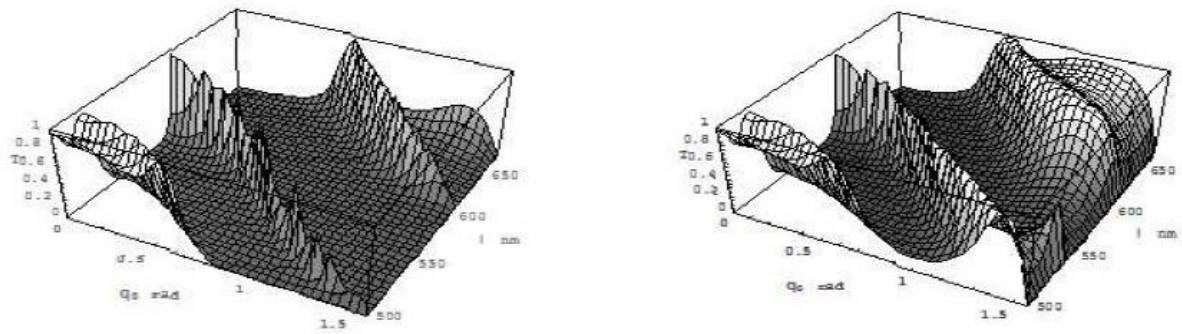

Figure 8. Stereogram of the TE wave defect pattern with incident angle and incident wavelength variation

Figure 9. Three-dimensional diagram of TM wave defect mode with incident angle and incident wavelength variation

Figure above showed that there is a very sharp protrusion is formed in the band gap in the both TE wave and the TM wave, forming a very narrow conduction band. Figure 10 showed obviously that the defect mode of doped photonic crystals.

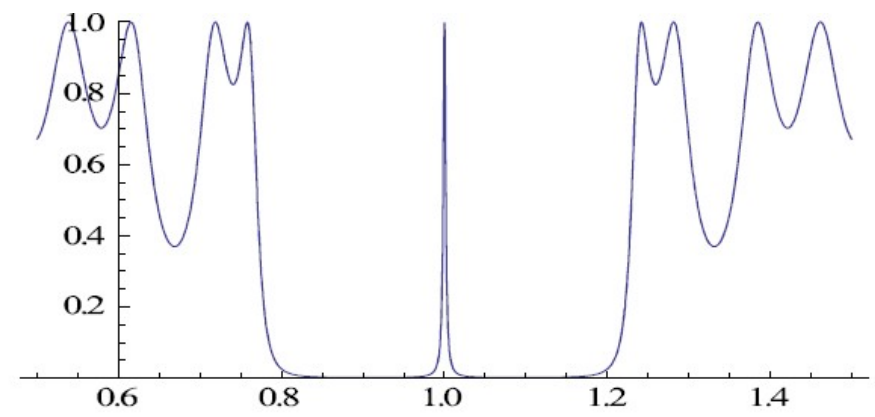

Figure 10. The Variation of Elastic Wave Defect Mode with Frequency

Previous research literature showed that the frequency of the one-dimensional doped photonic crystal defect mode is determined by the thickness of the doped medium. The frequency width of the defect mode is determined by the thickness and the refractive index of the dielectric film.

\section{The total reflection tunneling properties of photonic crystals}

The total reflection tunneling of one-dimensional photonic crystals is a new phenomenon found in the last two years. In order to obtain the total reflection tunneling of one-dimensional photonic crystals, the response curve of the transmittance of the light wave from the zinc sulfide to the single interface of magnesium fluoride with the incident 
angle is observed first. Since the refractive index of zinc sulfide is greater than the refractive index of magnesium fluoride, thus the light on the single interface will appear full reflection phenomenon, the total reflection angle is.Figure 7 showed that the transmittance decreases with the increase of the incident angle when the incident angle is less than the total reflection angle, and the transmittance decreases to 0 rapidly when the incident angle approaches the full reflection angle. When the angle of incidence is greater than the full reflection angle, the transmittance is always zero, that is, the full reflection phenomenon occurs, and the light cannot enter the magnesium fluoride from the zinc sulfide in the case of greater than the total reflection angle.
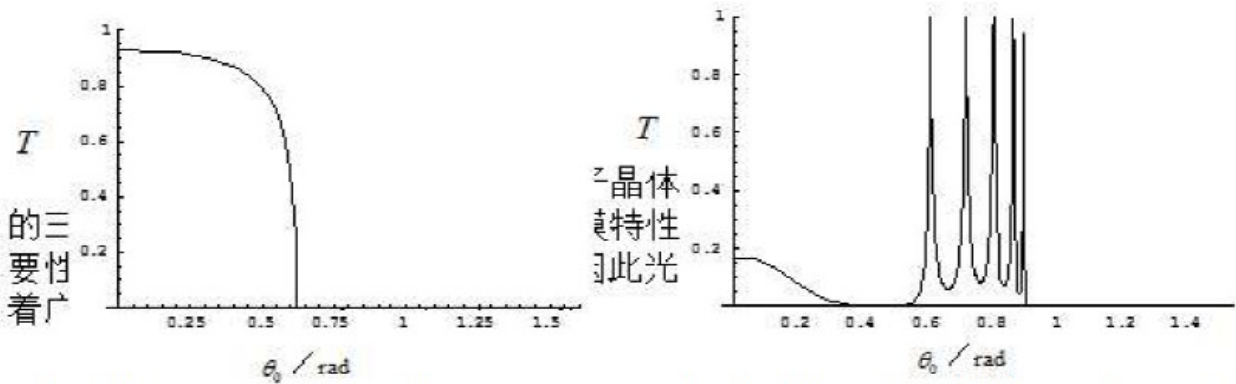

Figure 11. 7 The response curve of the transmittance at the single interface with the incident angle

Figure 12. Curve of the total reflection of the photon crystal with the incident angle

Further study is to determine the case of light incident in one-dimensional photonic crystals, that are alternately arranged by magnesium fluoride and zinc sulfide. The medium of the incident space and the exit space is also zinc sulfide. In this case, when the light is incident on the one-dimensional photonic crystal, the total reflection angle is. The response curve of the transmittance of the one-dimensional photonic crystal with incident angle is calculated as shown in Fig 11. When the incident of light on the one-dimensional photonic crystal, there are five obvious transmission peaks in the range of the angle of incidence which is greater than the full reflection angle, which indicates that the light wave can penetrate photonic crystals when the light wave is larger than the full reflection angle in photonic crystals. This phenomenon is called 'the total reflection tunneling effect of photonic crystals'.

It is also found that the number of total reflection tunneling peaks of one-dimensional photonic crystals is related to the number of cycles of one-dimensional photonic crystals, and the frequency of total reflection tunneling peaks decreases with the increase of the periodic optical thickness of photonic crystals. The discovery of the total reflection tunneling effect of photonic crystals has opened up a new research topic for photonic crystal research. And the total reflection tunneling peak of the photonic crystal has excellent comb filter characteristic, which provides a theoretical basis for designing a new high quality photonic filter by using the total reflection tunneling effect of photonic crystals.

The concept of photonic crystal and the commonly used research method of photonic crystal are introduced. Three important characteristics of one-dimensional photonic crystal are discussed, namely band characteristic, defect mode and total reflection tunneling. The use of these important properties of photonic crystals can effectively control the transmission of photons. Therefore, photonic crystal has a wide application prospect in optical signal transmission and optical signal control.

\section{Chapter 2: A research method of photonic crystal theory - feature matrix method}

The most important characteristic of photonic crystals is the photonic band gap, the optical materials and devices such as optical fiber, optical waveguide, full reflection mirror, filter, polarizer and so on are applying this characteristic where the photons cannot propagate through the band gap. When the defective medium is added to the photonic crystal, the propagation of the photon in the photon crystal can be localized to suppress or enhance its spontaneous radiation, so that the photonic crystal can be used to produce high efficiency and zero threshold laser, high quality laser resonator and efficient light-emitting diodes. However, to achieve the application of photonic crystals in these fields, we must know the band structure of photonic crystals, to know its band structure, we must select the appropriate research methods. At present, there are three main methods for the study of photonic crystal band structure: feature 
matrix method, plane wave expansion method, multiple scattering method and so on.

\section{The method of propagation of the photon during medium absorption}

For the media with absorption, in order to simultaneously describe its refraction and absorption of light waves, it has to be introduced the concept of complex refractive index and complex wave number:

$$
\hat{n}=n(1+i k) \quad 、 \quad \hat{k}=\frac{\omega}{c} \hat{n}
$$

Where is the refractive index of the medium to describe its refraction to the light, is the extinction coefficient of the medium to describe its absorption to the light, $\mathrm{c}$ being the speed of light in the vacuum, and is the circular frequency of the light. The light waves propagating in the absorption medium satisfy:

$$
\begin{aligned}
& \nabla^{2} \vec{E}+\hat{k}^{2} \vec{E}=0 \\
& \vec{E}=\vec{E}_{0} e^{i(\hat{\vec{v}} \cdot \vec{s}-a t)}
\end{aligned}
$$

is the unit vector for the direction of light propagation. Combined with formula (1):

$$
\vec{E}=\vec{E}_{0} e^{\frac{-\omega}{c} n \vec{r} \bullet \vec{s}} e^{i \omega\left(\frac{n}{c} \cdot \vec{r}-i\right)}
$$

It can be seen from (4) that the light wave propagating in the absorption medium is the attenuation wave. However, it can be seen from (2) and (3) that the equation and the corresponding solution in the absorption medium satisfy the equation and the corresponding solution in the transparent medium is exactly the same, but only the number of complex waves replaces the wave number. Therefore, in dealing with the propagation of light in the absorption medium, it is only necessary to change the refractive index in the formula corresponding to the transparent medium to the complex refrac- tive index, and the wave number can be solved by changing the number of waves.

2) the derivation of the characteristic matrix method

According to the thin film optics theory, we can use a $2 \times 2$ matrix to represent the transmission properties of light in each layer of the medium, which becomes the characteristic matrix of light propagation. For the first layer of media, the characteristic matrix is:

$$
M_{j}=\left[\begin{array}{cc}
\cos \delta_{j} & -\frac{i}{p_{j}} \sin \delta_{j} \\
-i p_{j} \sin \delta_{j} & \cos \delta_{j}
\end{array}\right]
$$

Where $=(2 /)$ cos,is the optical thickness of the dielectric layer andis the angle of light between medium layer and the normal direction of the interface, andis the wavelength of the incident light. Among them

$$
\begin{cases}p_{j}=\begin{array}{l}
n_{j} \cos \theta_{j} \\
\cos \theta_{j} / n_{j}
\end{array} \text { TII 波 }\end{cases}
$$

(1) The overall characteristic matrix of one-dimensional photonic crystals is:

$$
M=\left[\begin{array}{ll}
M_{11} & M_{12} \\
M_{21} & M_{22}
\end{array}\right]=\left(M_{1} M_{2}\right)^{N}
$$


When the light from the air emit into the photonic crystal, the reflection coefficient is:

The reflectance:

$$
R=|r|^{2}
$$

$$
T=1-R
$$

The transmittance.

(2) The overall characteristic matrix of one-dimensional doping photonic crystals is:

$$
M=\left[\begin{array}{ll}
M_{11} & M_{12} \\
M_{21} & M_{22}
\end{array}\right]=\left(M_{1} M_{2}\right)^{N} M_{3}\left(M_{2} M_{1}\right)^{N}
$$

When the light from the air emit into the photonic crystal, the reflection coefficient is:

$$
r=\frac{\left(M_{11}+M_{12} p_{h}\right) p_{0}-\left(M_{21}+M_{22} p_{h}\right)}{\left(M_{11}+M_{12} p_{h}\right) p_{0}+\left(M_{21}+M_{22} p_{h}\right)}
$$

The reflectance:

$$
T=1-R
$$

The transmittance of one-dimensional photonic crystals can be calculated by using the above formula, and thus the studies on bandgap and filter characteristics can be carried out.

\section{Chapter 3: The research on the influence of absorbing material on one-dimensional photonic crystal TM wave energy band}

\section{1. one-dimensional photonic crystal structure and light propagation characteristics}

One-dimensional photonic crystals are photonic crystal materials with periodic distribution of dielectric refractive index in one direction of space. The basic structure of a one-dimensional photonic crystal is composed of two kinds of dielectric films with different refractive indices. This structure makes the dielectric constant of photonic crystal which perpendicular to the dielectric layer periodically changes with the spatial position, whereas the dielectric constant which parallel with the dielectric layer is constant value. In this paper, we focus on the study of simple structure of one-dimensional photonic crystal, the structure is alternating composed of two types of material with the refractive index of and the thickness are and, respectively, and the thickness of a cycle is, this structure is similar with the multilayer dielectric film, The spatial refractive index is when the incident light wave is perpendicular to the photonic crystal interface, it is as shown in Figure 13:

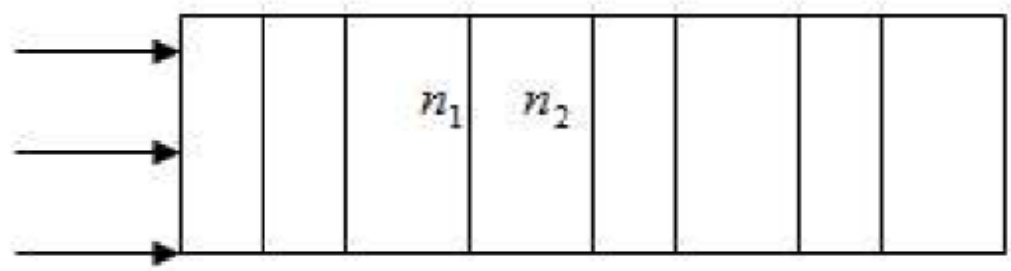

Figure 13. The structure of one - dimensional photonic crystals

When the light is propagated inside it, the bandgap and conduction band will appear, and this is the propagation characteristics of the simple structure of the one-dimensional photonic crystal and it also the main point of this paper. 


\section{Influence curve of the TM wave absorption band with the changes of frequency (2D)}

When $=1.38$ (magnesium fluoride), $=2.38$ (zinc sulfide) and the incident angle is $\theta=0.5 \mathrm{rad}$, the condition of the elimination coefficient, $\mathrm{k}$ at $0,0.01,0.03$ was observed when reflectivity, $\mathrm{R}$ changes with frequency, $\mathrm{g}$.

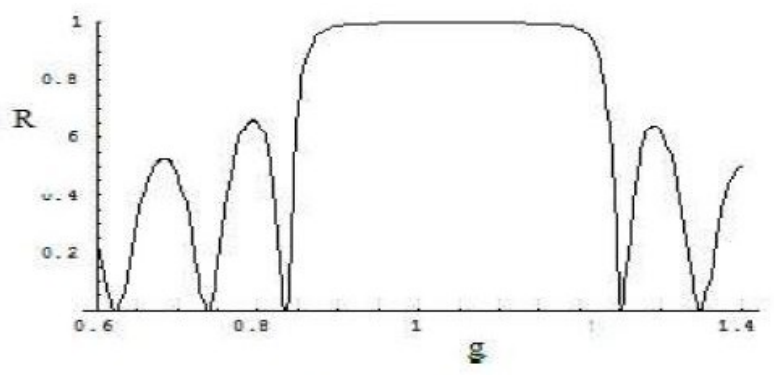

Figure 14. $\mathrm{k}=0$

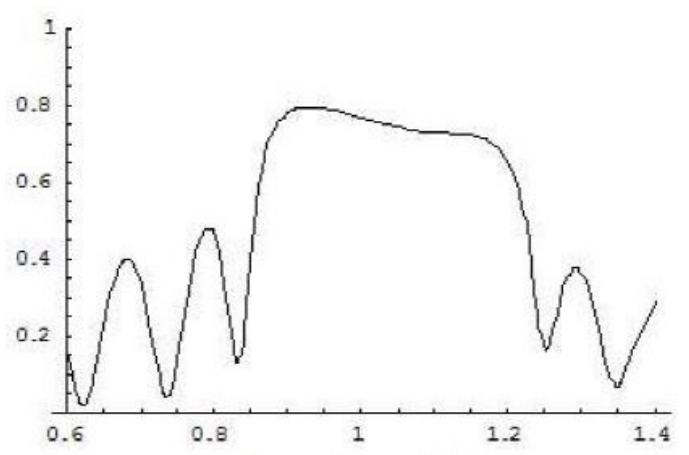

Figure 15. $\mathrm{k}=0.01$

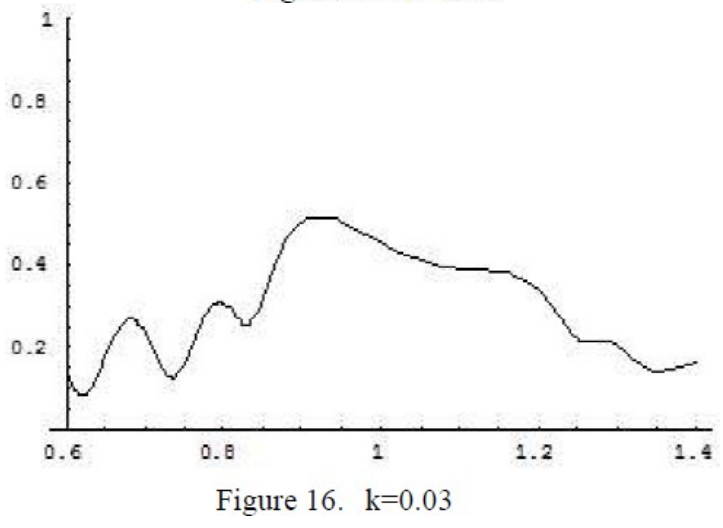

The figure above showed that when $\mathrm{K}=0.00$ (Figure 3.2), that is, there is no absorption at this time, the reflectivity $\mathrm{R}$ is 1 when frequency is in interval of $0.8-1.2$, then a band gap is appear, and the band distribution is very average.

When $\mathrm{k}=0.01$ (Figure 3.3), the band gap reflectivity decreases, the peak is about 0.8 , the band is still obvious. When $\mathrm{k}=0.03$ (Figure 3.4), the peak of the reflectivity dropped to about 0.6 , the band gap is no longer obvious and loss its function.

According to the above data, it showed that with increased of the absorption coefficient $\mathrm{k}$ value, the band gap will become weaker, the band gap reflection gradually reduced, the peak becomes smaller, the width also becomes narrow, and the peak almost disappeared at last.

\section{Influence curve of TM wave absorption band with changes of the absorption coeffcient and frequency (3D)}

When $=1.38$ (magnesium fluoride), $=2.38$ (zinc sulfide), the changes condition when the incident angle is $\theta$, the reflectivity $\mathrm{R}$ when the absorption coefficient, $\mathrm{k}$ is in the range of $(0-0.1)$, the frequency, $\mathrm{g}$ is in the range of (0.6-1.4) 
was observed. The experiment was carried on at $\theta=0,0.5,1,1.5 \mathrm{rad}$.
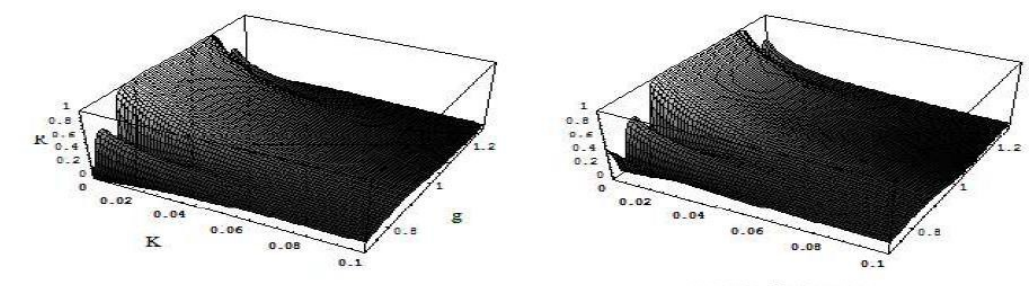

图 $3.5 \theta=0$

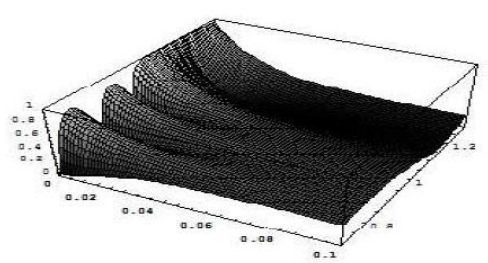

图 $3.7 \quad \theta=1 \mathrm{rad}$

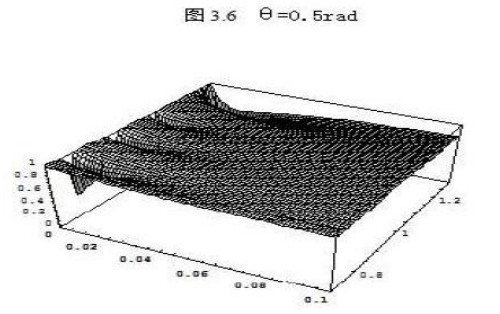

图 $3.8 \quad \theta=1.5 \mathrm{xad}$

The above figures were analyzed and showed that when $=0$ (no absorption), the reflectance of TM wave is also 1 when incident angle, $\theta$ is $0,0.5,1,1.5 \mathrm{rad}$, the band gap appear in all angle. When $\mathrm{k}$ increases to 0.05 , the reflectivity decreases to about 0.41 , the top of the band gap is not smooth, the edge is also blurred.

When $\theta=0$, the band gap is clear, the edge changes are very steep and obvious, and the peaks on both sides have the same trend, and when $\theta=0.5 \mathrm{rad}$ and $1 \mathrm{rad}$, although a clear band gap was still observed, but in interval od 1-1.4 of normalized frequency, the band edge gradient slowed down, it almost no changes can be seen, when $\theta=1.5 \mathrm{rad}$, the reflectivity changes more and more gentle, the peak almost unchanged.

When $\mathrm{k}$ is gradually increased, the corresponding transmission peak value of TM wave are also gradually increased when incident angle $\theta$ is $0,0.5 \mathrm{rad}, 1 \mathrm{rad}, 1.5 \mathrm{rad}$. As $\mathrm{k}$ increases gradually, the width of the band gap frequency decreases. Regardless of how the $\mathrm{k}$ and the angle of incidence change, the peak position is at the position of frequency $\mathrm{g}=1$.

When $\mathrm{k}$ is constant, the transmission peak increases with the increased of the incident angle, and the band gap reflectivity increases with the increased of the incident angle, and the frequency width decreases with the increased of the incident angle.

\section{Influence curve of TM wave absorption band with the cycle thickness and frequency changes (3D)}

When the changes was observed the incident angle is $\theta=0.5 \mathrm{rad}$, the absorption coefficient $\mathrm{k}$ is changed, the reflectivity $\mathrm{R}$ varies with the cycle thickness $\mathrm{X}(0.8-1.2)$, and the frequency $\mathrm{g}$ is (in the range of $=0.5-1.5) \mathrm{rang}$. The experiment was carried on at $\mathrm{k}=0,0.01,0.02,0.03$.

The figure showed that when $\mathrm{k}=0$, the reflectivity peak corresponding reflectance is 1 , and a very obvious photonic bandgap occurs at the frequency of $0.8-1.2$, and the width of the bandgap frequency is the largest.

- When $\mathrm{k}=0.01$, the reflectivity peak corresponding reflectance is reduced to about 0.8 in the frequency of $0.8-1.2$, and the photonic bandgap is still more obvious.

- When $\mathrm{k}=0.02$, the reflectivity peak corresponding reflectance is reduced to about 0.6 in the frequency of $0.8-1.2$, although it can see the photonic band gap, but it has begun to become blurred.

- When $\mathrm{k}=0.03$, the reflectivity peak corresponding reflectance is reduced to about 0.5 in the frequency of $0.8-1.2$, and the band gap of the photonic crystal has disappeared. 
From the above analysis, it can be concluded that when the $\mathrm{k}$ is gradually increasing, the reflectivity is decreased, the peak is getting lower and lower, and the reflectivity corresponding to the band gap is getting lower and lower. When $\mathrm{k}$ is constant, with the increase of the cycle thickness, the reflectivity decreases gradually, the corresponding reflectance peak is getting lower and lower, the band gap frequency range becomes more and more narrow.
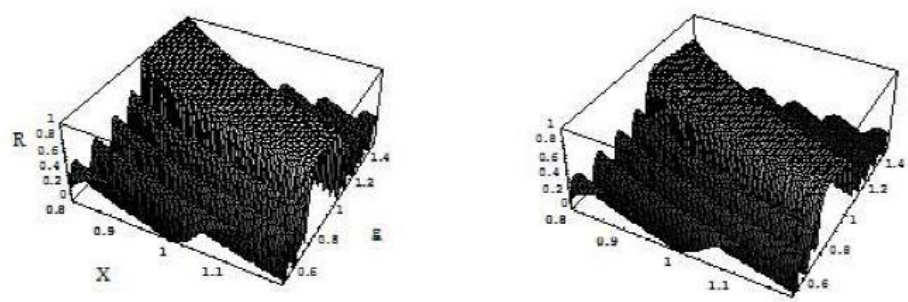

图 $3.9 \mathrm{k}=0$

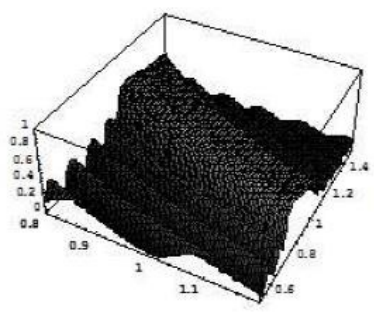

图 $3.11 \mathrm{k}=0.02$
图3.10 $\mathrm{k}=0.01$

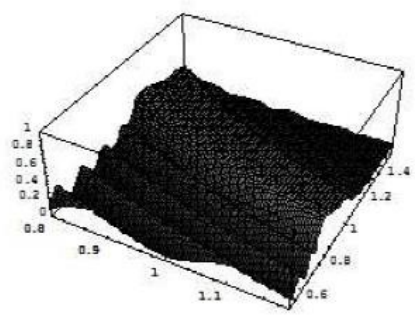

图 $3.12 \mathrm{k}=0.03$

\section{5. research conclusions}

By changing the incident angle and the absorption coefficient, the three-dimensional 3D image of the band of the onedimensional photonic crystal TM wave with the absorption coefficient and the cycle thickness and frequency is plotted by Wolfarm Mathematica software. Through the comparison analysis of the 3D image, the influence of the absorption coefficients on the energy band of the one-dimensional doped photonic crystal is as follows:

1. The extinction coefficient of the impurity has a significant effect on the transmission peak of the TM wave. Absorption coefficient $\mathrm{K}=0$, that is, no absorption state, the transmission is most obvious, the reflectivity, $\mathrm{R}=1$, there is a band gap, its shape similar to the rectangular, band gap waveform is more obvious and symmetrical. With the increase of the absorption coefficient $\mathrm{k}$, the band gap will become weaker and weaker, the band gap reflection will gradually decrease, the peak becomes smaller and the width will narrow, and finally the peak will disappear almost.

2. When the extinction coefficient increases to 0.03 , the corresponding peak reflectivity decreases, the band gap becomes narrows, the top is not, the future bandgap gradually disappear.

3. When the absorption coefficient $\mathrm{k}$ increases, the reflected wave width increases and the half width is gradually reduced.

4. When the extinction coefficient is constant, the transmission peak moves towards to the direction of high frequency with the increase of the incident angle, and when the incident angle changes, the peak of its reflectance is always 1 .

5. When the extinction coefficient is constant, the transmittance decreases with the increase of the cycle thickness, and the reflection peak also decreases with the increase of the cycle thickness, and the band gap is gradually disappears.

\section{References}

1. Zhenghai Tang. Modification of graphene and polymer / graphene composites, South China University of Technology, 2014, 4.

2. Novoselov K. S., Geim A. K., Morozov S. V., et al. Electric field effect in atomically thin carbon films [J]. Science, 2004, 306(5296): 666-669.

3. Qingkai Wang. Preparation of graphene and conductive properties of the Beijing Institute of Printing, $2014,12$. 
4. Wencai Ren, Libo Gao, Laipeng Ma, et al. Preparation of graphene by chemical vapor deposition [J]. New Carbon Materials, 2011, 26(1): 71-80.

5. Xiaoyang Hu. Preparation and application of carbon nanotubes and graphite dilute. Zhengzhou University, 2013(5).

6. Wenjun Kang. Controllable Synthesis, Characterization and Properties of Carbon-based Carbon Composites, University of Science and Technology of China, 2011, 4.

7. Berger C., Song. M., XB Li, XS. Wu, Brown, N, Naud C, W.A deHeer, et al. Science, 2006(312): 1191.

8. Campos L. C., Manfrinato V. R., Sanchez-Yamagishi J. D., et al. Anisotropic etching and nanoribbon formation in single-layer graphene [J]. Nano Letters, 2009, 9(7): 2600-2604.

9. Dongjie Li. Carbon-based composite materials preparation and performance research, Tianjin University, $2009,4$.

10. Xiaoyong Hu. Preparation of carbon-based composites and their electrochemical energy storage properties, Hunan University, 2013,9.

11. Haibo Li. Synthesis, characterization and characterization of carbon and carbon-based composites, China University of Science and Technology, 2010, 4.

12. Dong Guo. Graphite alkyne in the application of dye-sensitized solar cells. Qingdao University of Science and Technology, 2014, 4.

13. Huixue Li, Xiaofeng Wang, Zhifeng Li, et al. Theoretical study on the structure and properties of graphite [J] Acta Chimica Sinica, 2013,75-80.

14. Yongjun Li, Yuliang Li. Two-dimensional polymer - new carbon allotrope-like graphite alkyne [J]. Acta Polymerica Sinica, 2015,2 (2): 147-163. 